\title{
Optimization and Simulation of humidity sensor readout circuitry using two stage op amp
}

\author{
Nidhi Kuriyal $^{1}$, Rajeev Kumar ${ }^{2,}$ Vishal Ramola ${ }^{3}$ \\ M.Tech. Student VLSI Design Department UTU, Dehradun ${ }^{l}$, \\ Assist. Prof. Electronics Department UCST Dehradun ${ }^{2}$, \\ HOD. ECE Department UTU, Dehradun ${ }^{3}$
}

\begin{abstract}
In this paper a humidity sensor readout circuitry using two stage opamp is designed. This circuitry is realized in TSMC 0.18um CMOS Technology. Designing of two-stage op-amps is a multi-dimensionaloptimization problem where optimization of one or more parameters may easily result into degradation of others. The OPAMP circuit is designed to exhibit a unity gain frequency of $2.02 \mathrm{GHz}$ and exhibits a gain of $49.02 \mathrm{~dB}$ with a 60.50 phase margin and CMRR 39dB, Slew Rate 154dB, UGB (Unity Gain Bandwidth) are in a higher unity gain frequency under the same load condition. Also presented humidity sensor can achieve good linearity over the relative humidity range from $20 \%$ to $80 \%$. The total power dissipation of readout circuitry is $21.8 \mathrm{pW}$.
\end{abstract}

Terms: CMOS humidity sensor, humidity sensor, Op-Amp.

\section{Introduction}

Humidity sensors have a widely used in application areas, including agriculture, climate control, food storage, and domestic appliances. In order to satisfy the requirements of these applications, humidity sensors should provide high sensitivity over a wide range of humidity and temperature and linear response. Other important parameters of the humidity sensors can be listed as long-term stability, response time, and power consumption. A desirable feature of the humidity sensors is their compatibility with standard IC fabrication technologies. There are various types of humidity sensors based on the sensing principle they use, such as resistive, mechanical, gravimetric, capacitive, and thermal humidity sensors. This approach provides a number of advantages. The diodes can be heated up with a low power, and they provide high sensitivity. As the diodes are heated up, no water condensation can occur on the sensing elements.

In most of the electronics circuits the Operational Amplifiers is the most common building blocks. So as the transistor channel length and power supply is reduced then the design of Op amps face continuous challenge. Due to different aspect ratio (W/L), there is a tradeoff among speed, gain, power and the other parameters. The implementation of a CMOS OPAMPs that combines a considerable dc gain with higher unity gain frequency has been a most difficult problem. There has been several circuits design to evaluate this problem. The purpose of the design methodology in this paper is to design accurate equations for the design of high- gain 2 staged CMOS op-amp.

\section{Outline of paper}

This paper is organized as follows. Section II- presents the two stage amplifier. Section III- Differential transconductance amplifier and Modeling of sensor diode. Section IV- simulation result of two stage op amp and truth table. Section V- design and simulation of humidity sensor readout circuitry.

\section{Two Stage Cmos Op-Amp}

The various topologies of a new genus have evolved and been used in applications. Here, we chose a simple pair differential amplifier Immune input amplifier, common source amplifier (high gain) to the output amplifier, a current mirror circuit as bias circuit, and a buffer circuit compensation current as well as a Miller capacitance in series with one another.

For every iteration the entire design procedure above is carried on until the design is simulated for the specifications. For simplicity, only the $\mathrm{W} / \mathrm{L}$ ratios of transistor in the if an iteration are presented. The value of the compensating capacitor $\mathrm{Cc}=4 \mathrm{pF}$ to accommodate a variation of $+/-25 \%$

and still give a phase margin greater than $60^{\circ}$ since $\mathrm{Cc}>0.22 * \mathrm{C}_{\mathrm{L}}$

This topology of the circuit is that of a standard CMOS op-amp. It comprised of three subparts, namely differential gain stage, second gain stage and biasing network. It was found that this topology was able to successfully meet all of the design specifications. 


\section{II.1. Differential Gain Stage}

The first subsection of interest is the differential gain stage which is comprised of transistor M1 to M4. In figure 1 transistor M1 and M2 are standard $\mathrm{N}$ channel MOSFET transistors which form the basic input stage of the amplifier. The gate of M1 is the inverting input and the gate of M2 is the non-inverting input. A differential input signal applied across the two input terminals will be amplified according to the gain of the differential stage. The transconductance of this stage is simply the transconductance of M1 or M2. M3 and M4 are the active load transistors of the differential amplifier. The current mirror active load used in this circuit has three distinct advantages. First, the use of active load devices creates a large output resistance in a relatively small amount of die area.

\section{II.2 Second Gain Stage}

The second stage is a current sink load inverter. The purpose of the second gain stage, as the name implies, is to provide additional gain, in the amplifier. Consisting of transistor M5 and M8, this stage takes the output from the drain of M2 and amplifies it through M5 which is in the standard common source configuration. Similar to the differential gain stage, this stage employs an active device, M8, to serve as the load resistance for M5. The gain of this stage is the transconductance of M5 times the effective load resistance comprised of the output resistance of M5 and M8. M8 is the driver while M7 acts as load.

\section{II.3 Bias String}

The biasing of the operational amplifier is achieved with only two transistors along with a current source. Transistor M6 and the current source supply a voltage between the gate and source of M7 and M8. Transistor M7 and M8 sink a certain amount of current based on their gate to source voltage which is controlled by the bias string. M6 is diode connected to ensure it operate in the saturation region. Proper biasing of the other transistors in the circuit is controlled by the node voltages present in the circuit itself shown in figure 1.

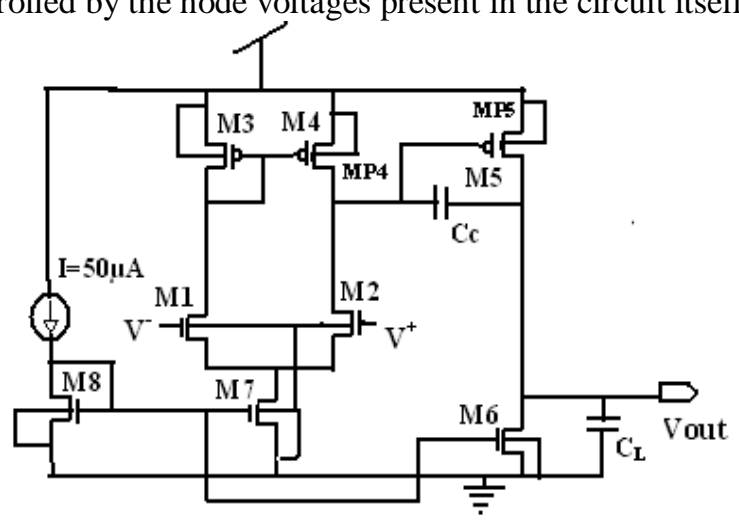

Figure.1. Two stage OPAMP with the compensation capacitor

Table-1: Transistor and size and the electrical parameters yielded of two stage op amp

\begin{tabular}{|c|c|c|c|c|}
\hline \multicolumn{3}{|c|}{ parameters } & \multicolumn{2}{|c|}{ The electrical parameters yielded } \\
\hline $\begin{array}{l}\text { Tran- } \\
\text { sistors }\end{array}$ & Length & Width & gmI, gmII & $806 \mu, 537 \mu$ \\
\hline Ml & $0.2 \mu \mathrm{m}$ & $15 \mu \mathrm{m}$ & Phase margin & $60.5^{0}$ \\
\hline M2 & $0.2 \mu \mathrm{m}$ & $15 \mu \mathrm{m}$ & $\mathrm{C}_{\mathrm{c}}$ & $24.8 \mathrm{fF}$ \\
\hline $\mathrm{M} 3$ & $0.4 \mu \mathrm{m}$ & $3.52 \mu \mathrm{m}$ & DC Gain & 53.40 \\
\hline M4 & $0.4 \mu \mathrm{m}$ & $3.2 \mu \mathrm{m}$ & Slew rate (+ve, -ve) & $154 \mathrm{~dB}$ \\
\hline M5 & $0.2 \mu \mathrm{m}$ & $6.2 \mu \mathrm{m}$ & CMRR & $39 \mathrm{~dB}$ \\
\hline M6 & $0.2 \mu \mathrm{m}$ & $1.6 \mu \mathrm{m}$ & Noise & $164 \mu \mathrm{V} / \mathrm{sqrt}(\mathrm{Hz})$ \\
\hline M7 & $0.2 \mu \mathrm{m}$ & $0.8 \mu \mathrm{m}$ & $\operatorname{PSRR}(+\mathrm{ve})$ & $154 \mathrm{~dB}$ \\
\hline M8 & $0.2 \mu \mathrm{m}$ & $0.4 \mu \mathrm{m}$ & Vdd & $3 \mathrm{~V}$ \\
\hline Iref & $50 \mu \mathrm{A}$ & & $\mathrm{C}_{\mathrm{L}}$ & $10 \mathrm{fF}$ \\
\hline & & & Power Consumption & $0.17 \mathrm{pW}$ \\
\hline
\end{tabular}

\section{Differential transconductance amplifier:}

Differential transconductance amplifier is used for converting the input differential voltage into current output which is integrated by the switch capacitor. The design is shown in figure 2 . The maximum differential voltage for which both the input transistor operate in active region is given by the following equation [4] 


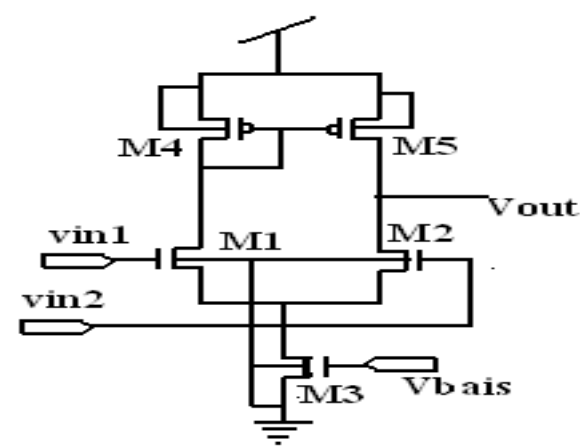

\section{III.1. Modeling of sensor diode}

Figure 2. Differential amplifier circuit

As given in [4] the thermal model of the sensor diode with the poly interconnects and air in between the diode and the substrate can be modeled as shown in the figure 3 .

$\mathrm{RT}_{\text {poly }}$ is the thermal conductivity of poly connecting the diodes to the read out circuit, $\mathrm{R}_{\mathrm{Tair}}$ is the thermal conductivity of air in between diode and the substrate, CT represents the thermal capacitance.

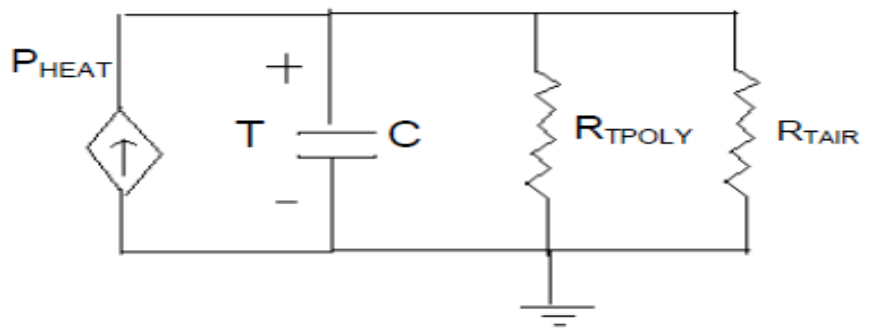

Fig 3: lumped element thermal circuit representing the self heating of a diode biased with constant current [4]

\section{Switched Capacitor (SC) integrator circuit}

The SC integrator correlated double sampling is used for offset compensation due to the finite gain effect of the operational amplifier [5, 6]. The schematic diagram of the switched capacitor (SC) integrator with the differential transconductance amplifier is as shown fig.6. The Sc integrator works in two non-overlapping phases' phil and phi2 as shown in fig 6 . The switches of the SC integrator are implemented using NMOS. When the clock phase phi1 is high, switches transistor MN3, MN4, MN12, and MN13 are on. In phase phi1 the bottom plate of capacitor $\mathrm{C} 1$ is charged to a voltage depending upon the current output from the differential transconductance amplifier through switch MN4 and the top plate of the capacitor $\mathrm{C} 1$ forced to ground potential through switch MN3. In phase phi1 the op-amp acts as a voltage follower as the switch MN13 is on. During phi1 high the right plate of capacitor C3 is at ground potential through switch MN12 and the left plate of the capacitor C3 charged to Voffset which is due to the finite gain of the operational amplifier. When phi2 is high switches MN4, MN6, and MN14 are turned on. During this phase the bottom plate of capacitor C3 forced to ground potential through switch MN6, so the inverting input of the op-amp is forced to a negative voltage which was previously present in the bottom plate of the capacitor during phase phi1 let us say Vs. Thus this negative voltage at inverting input of the op-amp swings the output strongly positive. But the input of the op-amp must be at virtual ground due to high gain of op amp. So the capacitive coupling back to the inverting input through $\mathrm{C}_{3}$ tends to pull the inverting input back towards zero. The circuit settles when the inverting node is once again at a virtual ground, which means that $C_{1}$ is now discharged. The positive charge $C_{1} V_{s}$ which was on the bottom plate of $\mathrm{C} 1$ has flowed to ground while the negative charge $\left(-\mathrm{C}_{1} \mathrm{Vs}\right)$ on the top plate of $\mathrm{C}_{1}$ has shifted to the left hand plate of $\mathrm{C}_{3} . \mathrm{C}_{1} \mathrm{Vs}$ on the top plate of $\mathrm{C}_{1}$ has shifted to the left-hand plate of $\mathrm{C} 3$. This requires that the right - hand plate of $\mathrm{C}_{3}$ have a charge $\left(+\mathrm{C}_{1} \mathrm{Vs}\right)$. However this charge must equal $\mathrm{C}_{3}$ Vout. Hence, we conclude that the output voltage swings to a value given by

$$
\text { Vout }=\left(\mathrm{C}_{1} / \mathrm{C}_{3}\right) \text { Vs }
$$

Offset voltage is compensated during phi2 because the left plate of capacitor $\mathrm{C}_{3}$ was initially charged to a voltage of Voffset.

\section{Simulation results of two stage op amp}

The design compensation strategy, the two-stage OPAMP in the figure was designed using the model parameter of tsmc0.18 micron CMOS process. The design parameters along with the electrical parameters yielded are as given in the table 1 . This circuit operates efficiently in a closed loop feedback system, while high bandwidth makes it suitable for high speed applications. The circuit operating conditions includes the room temperature as the operating temperature with a power supply of $3 \mathrm{~V}$ and a load of $10 \mathrm{fF}$. 
For the frequency response plot, an ac signal of $1 \mathrm{~V}$ is swept with 5 points per decade from a frequency of $10 \mathrm{KHz}$ to $4 \mathrm{GHz}$. Figure. 6 illustrates the frequency response which shows a dc gain in dB versus frequency in $\mathrm{Hz}$ (in log scale) and phase margin of OPAMP in open loop. The dc gain is found to be $49.02 \mathrm{~dB}$ and phase margin 60.50 which is good enough for an OPAMP operating at a high frequency. A unity gain frequency of $2.02 \mathrm{GHz}$ is excellent for an OPAMP when all the other parameters are also set at an optimized value.

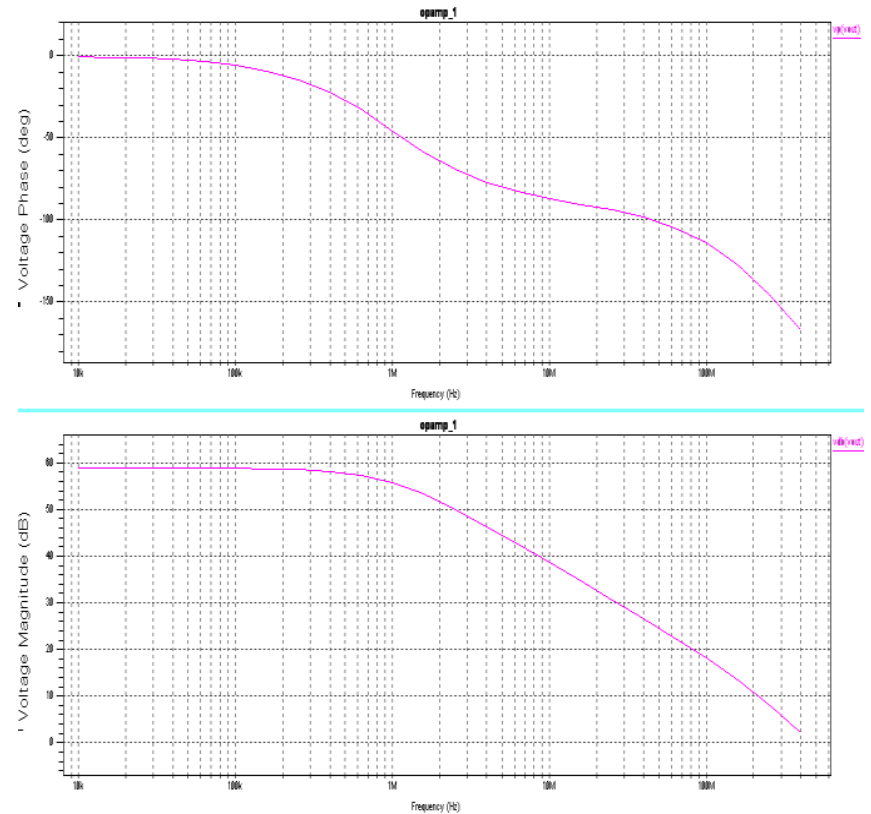

Figure.6: Frequency response of the OPAMP

The slew rate simulation is carried out performing a transient analysis using a pulse waveform of $1 \mathrm{mV}$ for a pulse period of $0.5 \mathrm{nsec}$. Slew Rate waveform ( $\mathrm{V}+$ is input wave and $\mathrm{V}$ - is output) and the calculated Slew Rate is $1.0758 \mathrm{~V} / \mathrm{uS}(+\mathrm{ve}$ slew rate) and $1.13 \mathrm{~V} / \mathrm{uS}(-\mathrm{ve}$ slew rate) respectively, which is quite good as compared to other low power, low voltage OPAMPs. The slew rate response is as shown below---

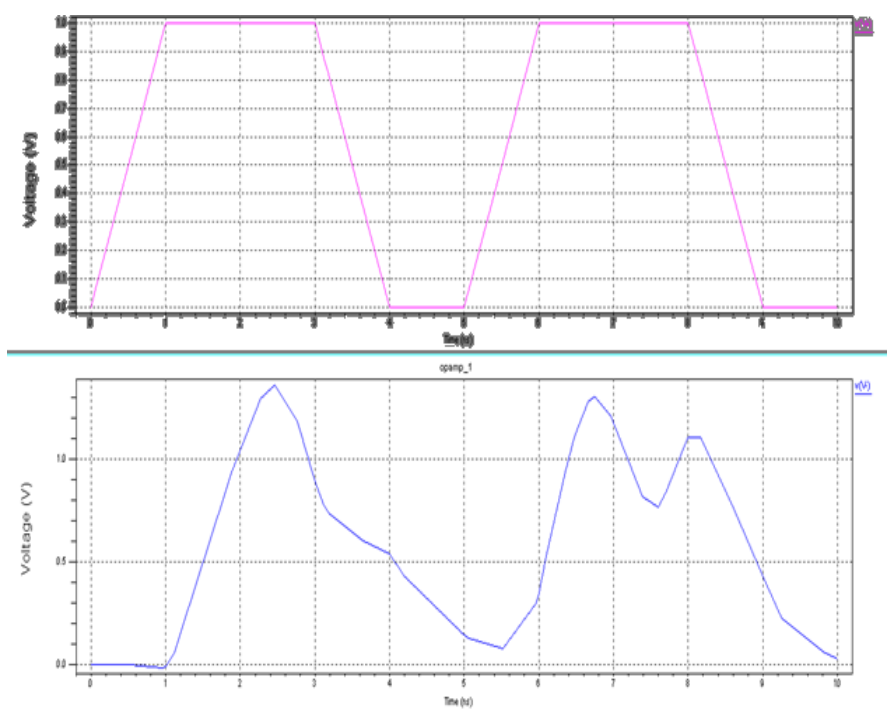

Figure.7. Slew Rate waveform

The simulate result of $\mathrm{CMRR}=39 \mathrm{~dB}$ 


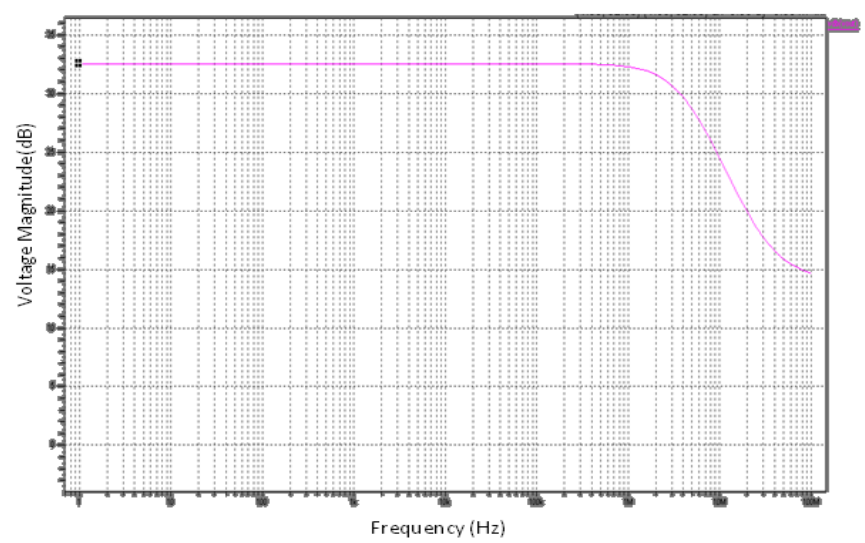

Figure.8. CMRR waveform

\section{VI-1. Results of Differential transconductance amplifier}

The operational amplifier and the differential transconductance amplifier are individually simulated using TSPICE in Tanner tool environment. The technology used for simulation is TSMC 180nm technology. The results are as shown in fig $6 \& 7$.The final transistor sizes in the design of op-amp are obtained after many numerical iterations and simulations. For example the design started with a bias current of $50 \mu \mathrm{A}$ and this resulted in low-gain, low phase margin and excess of band width. Hence the bias current is gradually scaled down for meeting the specifications by employing a bias current of $20+/-3 \mu$ against PVT variations. The value of compensating capacitor $\mathrm{Cc}=24.8 \mathrm{fF}$ has been taken to accommodate a variation of $+/-25 \%$ and still give a phase margin greater than $60 \mathrm{deg}$. since $\mathrm{Cc}>0.22 * \mathrm{C}_{\mathrm{L}}$. The supply voltage has been taken as $+/-10 \%$ i.e. 4.5 $\mathrm{V}$ shown in table 2 . The device length has been chosen as $180 \mathrm{~nm}$ to reduce the short channel effects and keeping channel length modulation constant [6].

The op-amp with the following constraints

i. voltage variation $+/-10 \%$ i.e. $4.5 \mathrm{~V}$

ii. Bias current variation $20+/-3 \mu \mathrm{A}$

iii. Temperature variation $20^{\circ} \mathrm{C}$ to $60^{\circ} \mathrm{C}$

iv. Compensating capacitor variation $+/-25 \%$

v. all the process corners (SS, SF, FF, FS, typical)

Table:2

\begin{tabular}{|l|l|l|l|l|l|}
\hline $\begin{array}{l}\text { Worst Case } \\
\text { Analysis }\end{array}$ & $\begin{array}{c}\text { Bias Current } \\
(\mu \mathrm{A})\end{array}$ & $\begin{array}{l}\text { Compensating } \\
\text { Capacitor(PF) }\end{array}$ & $\begin{array}{c}\text { Temperature } \\
\left({ }^{\circ} \mathrm{C}\right)\end{array}$ & $\begin{array}{l}\text { Voltage } \\
(\mathrm{V})\end{array}$ & $\begin{array}{l}\text { Process } \\
\text { Corner }\end{array}$ \\
\hline $\begin{array}{l}\text { IC.IR } \\
\text { (Input Common } \\
\text { Mode Range) } \\
=0 \text { to 2.85V }\end{array}$ & 17 & $5 \mathrm{f}$ & 20 & 4.5 & Fast-slow \\
\hline $\begin{array}{l}\text { PM(Phase } \\
\text { Margin)=60.5 }\end{array}$ & 17 & $5 \mathrm{f}$ & 60 & 4.5 & Slow-fast \\
\hline DC Gain=60 db & 17 & $5 \mathrm{f}$ & 60 & 4.5 & Slow-fast \\
\hline $\begin{array}{l}\text { SLEW RATE } \\
=1.078 v / \text { ssec }\end{array}$ & 17 & 5f & 60 & 4.5 & Fast-slow \\
\hline $\begin{array}{l}\text { UGB(Unity Gain } \\
\text { Bandwidth) } \\
=3.0970\end{array}$ & 17 & 5f & 60 & 4.5 & Slow-Fast \\
\hline
\end{tabular}




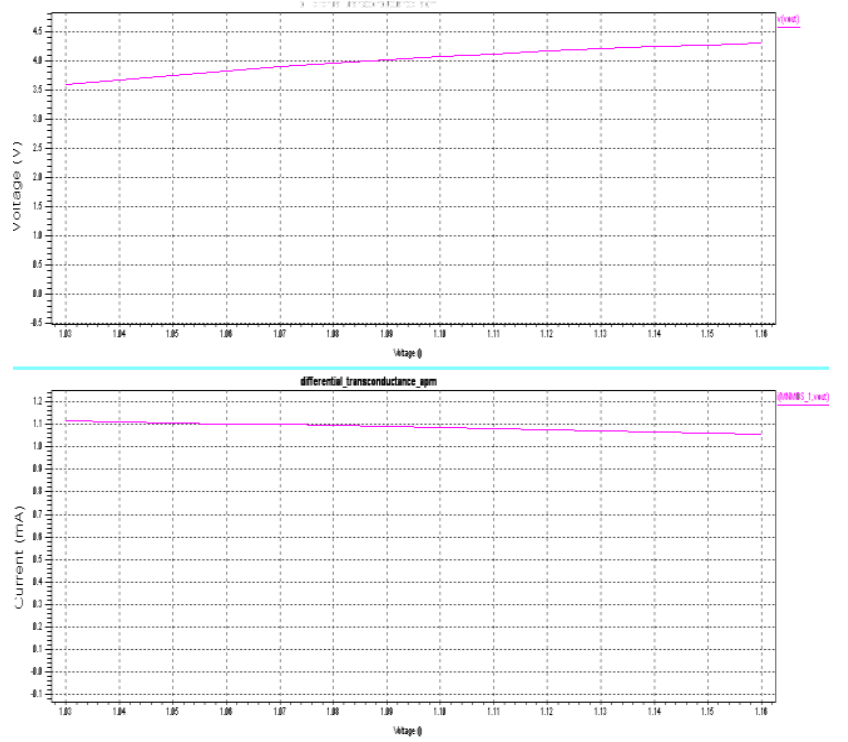

Figure 8: Waveform of Differential transconductance amplifier

\section{Conclusions}

In this paper, a humidity sensor read out circuitry using two stage opamp is designed. The results shows that the two stage operational amplifier optimized has successfully satisfied all the design specification given in advance. The Op-Amp specifications are met under all the process corners. The differential transconductance amplifier has also been designed for the required linear range of operation. The SC integrator and the differential transconductance amplifier are integrated and the output is verified by giving a constant voltage to the Non-inverting input and the sinusoidal voltage to the Inverting input. The design can be used for humidity sensor and can be adopted by the industries for its flexibility in design which would be beneficial from the point of view of production cost of industries.

\section{References}

[1]. B. Razavi, Design of Analog CMOS Integrated Circuits. McGraw-Hill, 2002

[2]. P.E. Allen and D.R. Holberg, CMOS Analog Circuit Design. Oxford University Press, 2002.

[3]. G. Palmisano, G. Palumbo and S. Pennisi "Design Procedure for Two-Stage CMOS Transconductance Operational Amplifiers: A Tutorial"; Analog Integrated Circuits and Signal Processing, 27, 179-189, 2001.

[4]. Burak Okcan and Tayfun Akin, "A Low-Power Robust Humidity Sensor in a Standard CMOS Process" IEEE TRANSACTIONS ON ELECTRON DEVICES, VOL. 54, NO. 11, NOVEMBER 2007

[5]. Dave Van Ess, "Understanding Switched Capacitor Analog Blocks", Application Note, cypress Microsystems 2004.11

[6]. Yunteng Huang, Paul Ferguson, and Gabor C. Temes, "Reduced Nonlinear Distortion in Circuits with Correlated Double Sampling", proc. IEEE int. symp. Circ. Syst. May 1996

[7]. G. Palmisano and G. Palumbo, "A compensation strategy for two-stage CMOS OPAMPs based on current buffer,". IEEE trans. Circuits sys.I, Fundam. Theory Appl., vol. 44, no. 3 , pp. 257- 262, mar1997.

[8]. J. Mahattanakul, "Design procedure for two stage CMOS operational amplifier employing current buffer", IEEE trans. Circuits sys. II, Express Briefs, vol 52, no.11 nov 2005

[9]. Mahattanakul, J. and Chutichatuporn, J. (2005) " Design Procedure for Two-Stage CMOS OPAMP with Flexible Noise-Power Balancing Scheme". IEEE Transaction on Circuits and Systems-I: Regular Paper 52, pp. 1508-1514.

[10]. Pugliese, A. , Cappuccino, G. and Cocorullo, G. (2008) " Design Procedure for Settling Time Minimization in Three-Stage Nested Miller Amplifiers". IEEE Transaction on Circuits and Systems-II 55, pp. 1-5.

[11]. Turchetti, C. and Masetti, G. (1983) "A Macromodel for Integrated All-MOS Operational Amplifiers". IEEE Journal of Solid-State Circuits SC-18, pp. 389-394.

[12]. Yang, H. C. and Allstot, D. J. (1990) "Considerations for Fast Settling Operational Amplifiers". IEEE Transactions on Circuits and Systems 37, pp. 326-334.

[13]. Yavari, M., Maghari, N. and Shoaei, O. (2005) "An Accurate Analysis of Slew Rate for Twostage CMOS Opamps". IEEE Transactions on Circuits and Systems - II: Express Briefs 52, pp. 164-167.

[14]. C. Makris and C. Toumazou, "Current-mode active compensation techniques, "Electron. Letters, vol. 26, no. 21, pp. 1792-1794, Oct. $11,1990$.

[15]. U. Kang and K. D. Wise, "A high speed capacitive humidity sensor with on-chip thermal reset," IEEE Trans. Electron Devices, vol. 47, no. 4, pp. 702-710, Apr. 2000.

[16]. R. Jachowicz and J. Weremczuk, "Sub-cooled water detection in silicon dew point hygrometer," Sens. Actuators A, Phys., vol. 85, no. 1-3, pp. $75-83$, Aug. 2000

[17]. Y. Y. Qui, C. Azeredo-Leme, L. R. Alcacer, and J. E. Franca, “A CMOS humidity sensor with on-chip calibration,” Sens. Actuators A, Phys., vol. 92 , no. $1-3$, pp. 80-87, Aug. 2001

[18]. B. Okcan and T. Akin, "A thermal conductivity based humidity sensor in a standard CMOS process," in Proc. $17^{\text {th }}$ IEEE Int. Conf. MEMS, Maastricht, The Netherlands, Jan. 2004, pp. 552-555. 


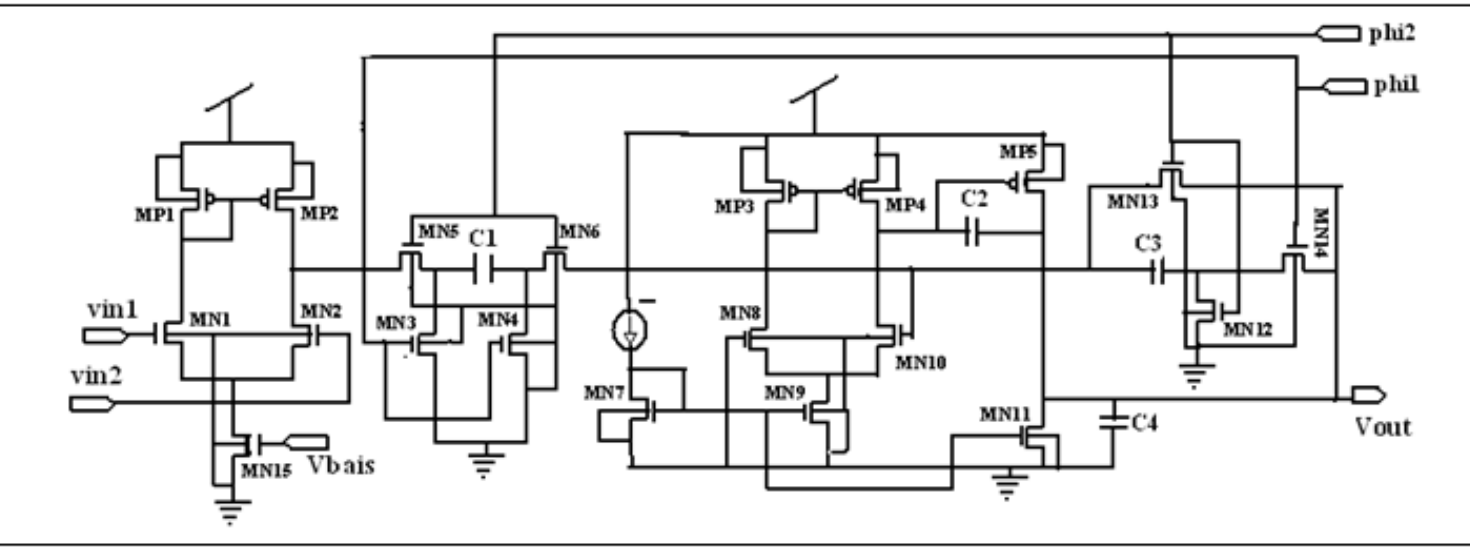

Figure 6: Schematic of humidity sensor with read out circuit

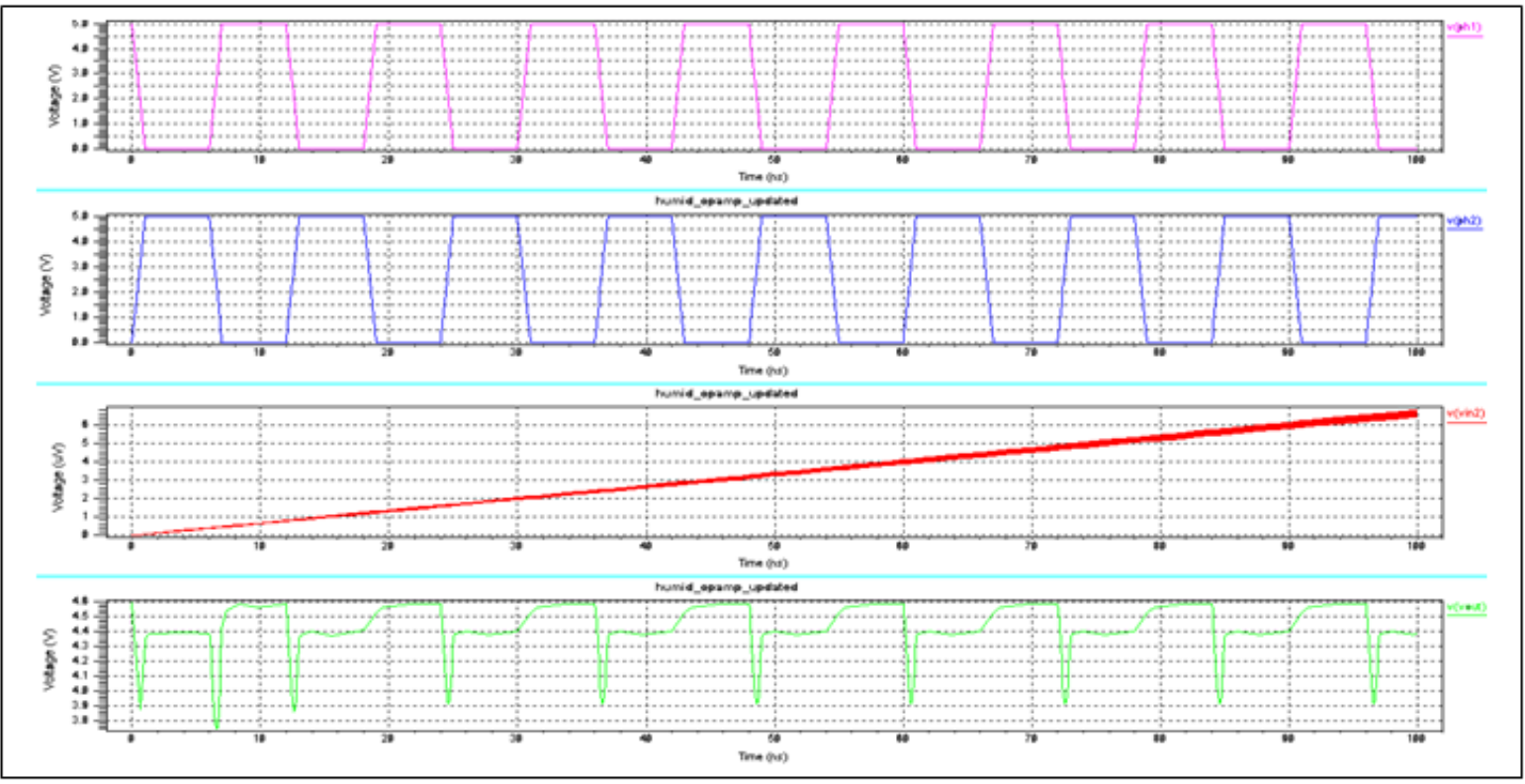

Figure 7: Waveform of humidity sensor with read out circuit 